\title{
CDISC CDASH Unit of Drug Dispensed or Returned Terminology
}

National Cancer Institute

\section{Source}

National Cancer Institute. CDISC CDASH Unit of Drug Dispensed or Returned

Terminology. NCI Thesaurus. Code C78421.

A terminology subset of the CDISC Study Data Tabulation Model (SDTM) Unit codelist created for Clinical Data Acquisition Standards Harmonization (CDASH) Unit of Drug Dispensed or Returned T erminology. 